\title{
Rolling Polyhedra on a Plane, Analysis of the Reachable Set*
}

\author{
Yacine Chitour, Alessia Marigo, Domenico Prattichizzo, Antonio Bicchi \\ Centro "E. Piaggio" \\ Università di Pisa
}

\begin{abstract}
The problem of dexterous manipulation of objects, i.e. of arbitrary relocation and reorientation of rigid bodies by action of some mechanism, is considered. We build upon previous results on the possibility of implementing dexterous "robot hands" with few actuators, which can be afforded through the exploitation of nonholonomic rolling of regular surfaces. In this paper we focus on the manipulation of polyhedral objects, and prove a necessary and sufficient controllability-like result, which discloses some of the interesting aspects and perspectives of this problem.
\end{abstract}

\section{Introduction}

The design of mechanisms for orienting and locating parts is of paramount importance in flexible automation and robotics. In some cases, the problem is that of reorienting a large number of parts coming in random positions and orientations, to a given posture within assembly tolerances. For such problems, industry most often uses ad hoc fixtures, such as vibrating part-feeders, fenced conveyor belts, etc.. The design and tuning of these devices is often time-consuming, and is only

\footnotetext{
${ }^{*}$ Work done with the partial support of ESPRIT W.G. LEGRO, contr. no. 032/94/TS. Yacine Chitour is currently a Visiting Scientist in Pisa with the support of ERNET, the European Robotics Network, EU(HCM) contr. no. ERBCHRXCT930381. Alessia Marigo is currently Visiting Scholar at the Institute d'Analyse Algébrique, Université de Paris 6
}

rewarding on large-size batch production. In other cases, where the typology of parts is more variate, more flexible manipulation means are preferable. In highly-flexible automation and robotics, the design of manipulation devices has been attacked by several different approaches, such as by developing dexterous multifingered hands ([Jacobsen et al., 1984], [Salisbury et al., 1985]); using "pushing" or "tilting" actions ([Peshkin and Sanderson, 1988], [Lynch and Mason, 1995]); "regrasping" ([Tournassoud et al., 1987], [Goldberg, 1993]); and "finger gaiting" ([Rus, 1992], [Chen and Burdick, 1993]).

Among these manipulation strategies, those using discontinuous contacts between the manipulator and the part are sometimes regarded as not reliable enough in real-world, unsteady environments. On the other hand, multifingered robot hands are often too costly, heavy, and complex, to be viable in many applications.

The idea of exploiting the nonholonomic nature of the constraint of rolling surfaces, so as to design a dexterous hand with few actuators, was presented in [Bicchi and Sorrentino, 1995]. In that paper, building upon previous results of Li and Canny [1990], it was conjectured that the position and orientation of an object with regular surface could be arbitrarily changed by rolling onto another regular surface by acting only on its relative angular velocities. The simple experiment of rolling a sphere on a plane surface, bringing it back to its initial position but with different orientation, is an intuitive explanation of this phenomenon. Based on 


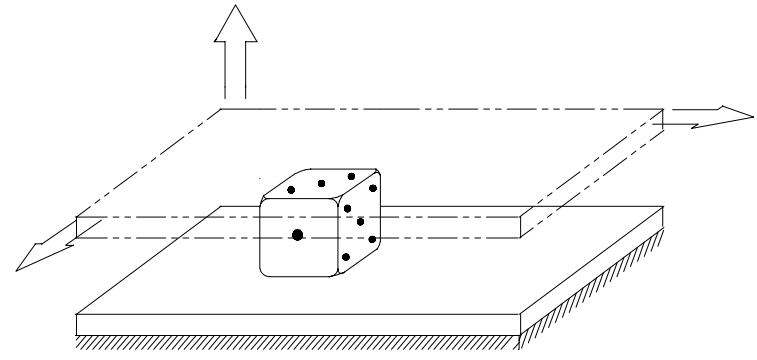

Figure 1: A parallel-jaw gripper can manipulate polyhedral parts

the above controllability conjecture, a dexterous hand consisting of two parallel plates, with only three translational degrees of freedom, was devised and experimentally demonstrated (see fig. 1). Bicchi, Prattichizzo, and Sastry [1995] confirmed later on the conjecture for all strictly convex objects with a regular surface of revolution, and discussed the differential-geometric aspects of planning and controlling the object motions.

The advantage of manipulation by rolling is that it accomplishes dexterity with very simple hardware, while it guarantees that the object is never "left alone" during manipulation. The intrinsic nonholonomic nature of rolling offers many difficulties to the planification and control of such devices, of which only few have been addressed so far.

Among the various open problems, the one we start considering in this paper is that of removing the limitation that manipulated objects should have regular $\left(C^{\infty}\right.$, analytic) surface. The main motivation of such an assumption is that for regular surfaces the powerful tools of differential geometry and nonlinear control theory are readily available. On the other hand, the assumption is rarely verified with industrial parts, which often have edges and vertices. Again, the simple experiment of rolling a die onto a plane without slipping, and bringing it back after any sufficiently rich path, shows that its orientation has changed in general, and hints to the fact that manipulation of parts with non-smooth (e.g., polyhe-

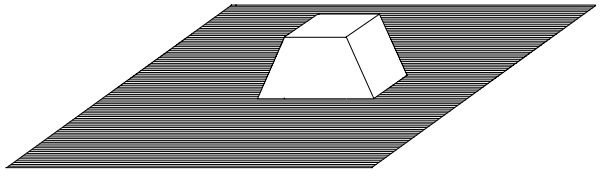

Figure 2: A polyhedron whose reachable set is everywhere dense

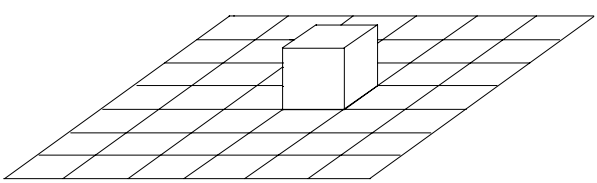

Figure 3: A polyhedron whose reachable set is nowhere dense

dral) surface can be advantageously performed by rolling.

Some aspects of graspless manipulation of polyhedral objects by rolling have been considered already in the robotics literature (see e.g. Sawasaki et al. [1989], Aiyama et al. [1993], Erdmann et al. [1991]). However, a complete study on the analysis, planning, and control of rolling manipulation for polyhedral parts is far from being available, and indeed it comprehends many aspects, some of which appear to be non-trivial. In particular, the lack of a differentiable structure on the configuration space of a rolling polyhedron deprives us of most techniques used with regular surfaces. Moreover, peculiar phenomena may happen with polyhedra, which have no direct counterpart with regular objects. In this paper, we start such study by analysing the structure of the set of configurations reachable from a given one, and show that it may reveal extremely different structure depending on the polyhedron considered (see fig. 2 and 3 ).

\section{Problem formulation}

Consider the simple device depicted in fig. 1, consisting of two plates, one of which is fixed, while the other can translate remaining paral- 
lel to the first. A part of known shape is put between the plates and successively moved by a combination of vertical and horizontal forces at the contacts that cause it to move. The goal is to bring the part from a given initial configuration (a point in $S E(3)$ ) to another desired one. A few considerations are in order:

- as the part is constrained to keep in touch with the two plates, to specify arbitrary desired configurations would require being able to move the lower plate vertically. With no loss of generality we only consider different configurations modulo a rigid translation of the whole mechanism;

- the surface of the part is considered to be piecewise flat, closed, and comprised of a finite number of faces, edges, and vertices;

- parts need not be convex. However, as the plates are assumed to be large w.r.t. the diameter of parts, we will only be concerned with the convex hull of the parts themselves. In what follows, we use the term "part" to refer to a piecewise flat convex surface, i.e., to a convex polyhedron;

- in general, three motions of a polyhedron on a plane are possible: by sliding on a face, tumbling about an edge, or pivoting about a vertex. Slippage is not considered desirable in this context, as it does not guarantee reliable enough results in manipulation. We assume therefore that high-friction, compliant materials are employed to cover the plates, and that the vertical degree-of-freedom of the upper plate is suitably used, so as to prevent slippage. By a similar concern, pivoting about a vertex is also banned. In fact, real-world parts never have perfectly sharp vertices, and the actual effects of pivoting about a vertex will strongly depend on the details of how "the corner is rounded" (recall that all the curvature of a polyhedron is concentrated at its vertices). On the other hand, tumbling about an edge is insensitive to the details of how the "edge" really looks like, since no curvature is concentrated on the edge of a polyhedron.

The only motions of the parts we will be concerned with are therefore comprised of a sequence of rotations about one of the edges of the face being in contact with the plate, by the amount that exactly brings another face in contact. This action on the parts will be referred to as an elementary tumble, or ET for short.

\section{Definitions and properties}

Let $\widetilde{P}$ be a convex polyhedron rolling on a plane $P$ by ET's. We associate to $\widetilde{P}$ the following sets:

(a) $\widetilde{V}=\left\{v_{1}, \ldots, v_{m}\right\}$ is the set of vertices of $\widetilde{P}$ and $m=\operatorname{card}(\widetilde{V})$;

(b) $\widetilde{E}=\left\{e_{1}, \ldots, e_{k}\right\}$ is the set of edges of $\widetilde{P}$ and $k=\operatorname{card}(\widetilde{E})$;

(c) $\widetilde{F}=\left\{F_{1}, \ldots, F_{l}\right\}$ is the set of faces of $\widetilde{P}$ and $l=\operatorname{card}(\widetilde{F})$.

By the assumption of convexity, parts are topological spheres, hence for their Euler characteristic it holds $\chi=m-k+l=2$.

The configuration space $\widetilde{M}$ of the system under investigation is the restriction of the space of rigid body configurations $S E(3)$ to those that have one face in contact with the plane $P$. One possible parameterization of this space is as follows.

Let $O x y$ be a fixed reference frame on the plane $P$. For each face $F_{i}, 1 \leq i \leq l$, let $c_{i}$ and $u_{i}$ be two arbitary distinct points fixed on $F_{i}$, for instance the center of gravity of $F_{i}$ and one of its vertices. Let $\left(x_{i}, y_{i}\right)$ be the coordinates of $c_{i}$, and $\theta_{i}$ be the oriented angle between $O x$ and $\vec{c}_{i} \vec{u}_{i}$. A configuration of $\widetilde{P}$ on $P$ is uniquely determined by the quadruple $\left(x_{i}, y_{i}, \theta_{i}, i\right)$, where $i \in\{1, \ldots, l\}$ is the index of the face in contact with $P$. The configuration set of our problem consists therefore of $l$ copies of $S E(2)$, or explicitly

$$
\widetilde{M}=\mathbb{R}^{2} \times S^{1} \times \widetilde{F} .
$$


For $1 \leq i \leq l$, each copy of $S E(2)$ corresponds to the set of all the possible configurations for the face $i, \widetilde{M}_{i}$, i.e.

$$
\widetilde{M}_{i}=\mathbb{R}^{2} \times S^{1} \times\{i\} .
$$

The space $\widetilde{M}$ is endowed with the product metric associated to the metrics of the euclidean space $\mathbb{R}^{2}$, of the quotient space $S^{1}=\mathbb{R} / 2 \pi \mathbf{Z}$ and of the discrete space $\widetilde{F}$, respectively. The latter is taken to be $\rho\left(F_{i}, F_{j}\right)=1-\delta_{i j}$, where $\delta_{i j}$ is the Kronecker symbol. Although very intuitive, this parameterization does not turn out to be the most convenient for our developments. We therefore introduce a slightly more technical description of $\widetilde{M}$ as the set of equivalence classes on a set $\widetilde{M}^{\prime}$ by the relation $\sim$, where

- the set $\widetilde{M}^{\prime}$ is defined as the subset of $\mathbb{R}^{2} \times$ $\widetilde{V} \times S^{1} \times \widetilde{F}$ of points $(x, y, v, \theta, i)$ where $i$ is the index of the face $F_{i}$ in contact with $P, v$ is any of the vertices of $F_{i}$ (shortly $\left.F_{i} v\right),(x, y)$ are the coordinates of $v$ and $\theta$ is the oriented angle between $\overrightarrow{x x^{\prime}}$ and $\overrightarrow{c_{i} v}$;

- two elements of $\widetilde{M^{\prime}}$ are equivalent under the relation $\sim$ if $i=i^{\prime}$ and $\theta^{\prime}-\theta$ is equal to the oriented angle between $\overrightarrow{c_{i} v}$ and $\overrightarrow{c_{i} v^{\prime}}$, for any fixed point $c_{i}$ on face $F_{i}$

Note that corresponding to each configuration of the polyhedron, we have an equivalence class with $n_{F_{i}}$ elements, where $n_{F_{i}}$ is the number of vertices of the face $F_{i}$.

The actions we take on the configurations of the polyhedron are finite sequences of ET's, that will be referred to as "trips". The length of a trip is the number of ET's it is comprised of. The problem this paper is concerned with is to understand the structure induced on the configuration space by trips of arbitrary length. We therefore define reachability of a configuration as

Definition 1 The configuration $q_{f}$ is reachable from $q_{0}$ if there exists a trip steering $\widetilde{P}$ from $q_{0}$ to $q_{f}$. In this case, we write $q_{0} \rightarrow q_{f}$.

For every $q \in \widetilde{M}$, let $\widetilde{R}_{q}$ be the reachable set from $q$, i.e. the set of configurations that can be reached from $q$ in a finite, but arbitrarily large number of ET's.

As mentioned in the introduction, the structure of the reachable set can be very diverse for different polyhedra. Note first that $\widetilde{R}_{q}$ is countable by its definition and therefore the inclusion

$$
\widetilde{R}_{q} \subset \widetilde{M}
$$

is strict. Introducing the canonical projections

$$
\begin{aligned}
& \Pi_{1}: \widetilde{M} \rightarrow \mathbb{R}^{2}, \\
& \Pi_{2}: \widetilde{M} \rightarrow S^{1},
\end{aligned}
$$

we have that $\Pi_{1}\left(\widetilde{R}_{q}\right)$ is trivially infinite and unbounded in $\mathbb{R}^{2}$. Various possibilities can occur: $\Pi_{1}\left(\widetilde{R}_{q}\right)$ (resp. $\Pi_{2}\left(\widetilde{R}_{q}\right)$ ) can be discrete in $\mathbb{R}^{2}$ (resp. finite in $S^{1}$ ), can have a finite or infinite number of points of accumulation in $\mathbb{R}^{2}$ (resp. idem in $S^{1}$ ) or can be dense in $\mathbb{R}^{2}$ (resp. idem in $S^{1}$ ). One can even distinguish differently dense structures for $\widetilde{R}_{q}$, among which are the following:

a) Density in $\widetilde{M}$ :

$$
(\mathbf{D M})\left\{\begin{array}{l}
\forall \varepsilon>0, \forall q_{f} \in \widetilde{M}, \\
\exists q^{\prime} \in \widetilde{R}_{q} \text { such that } \\
q^{\prime} \in B_{\varepsilon}\left(q_{f}\right),
\end{array}\right.
$$

b) Density in $\mathbb{R}^{2} \times S^{1}$ for a given face $i$ :

$$
(\mathbf{D M})_{i}\left\{\begin{array}{l}
\forall \varepsilon>0, \forall q_{f} \in \widetilde{M}_{i}, \\
\exists q^{\prime} \in \widetilde{R}_{q} \text { such that } \\
q^{\prime} \in B_{\varepsilon}\left(q_{f}\right) .
\end{array}\right.
$$

Here, $\mathbf{B}_{\varepsilon}(\cdot)$ indicates a ball centered in its argument of radius $\varepsilon$ in the suitable metric. Note that

$$
(\mathrm{DM}) \Rightarrow(\mathbf{D M})_{i}
$$

As usual, if the above properties hold for any initial configuration $q$, the properties will be said to hold globally.

In this paper, we explicitly consider two extreme cases of such behaviours represented in fig. 2 and 3, where we have respectively that

1. the reachable set is dense in $\widetilde{M}$ (see section 4); 


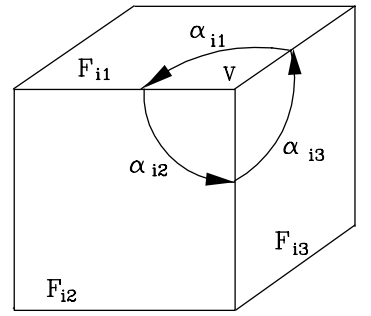

Figure 4: The relative angle at vertex $\mathrm{V}$ is defined as $\beta_{v}=2 \pi-\left(\alpha_{i 1}+\alpha_{i 2}+\alpha_{i 3}\right)$

2. $\Pi_{1}\left(\widetilde{R}_{q}\right)$ is a square lattice of $\mathbb{R}^{2}$ and $\Pi_{2}\left(\widetilde{R}_{q}\right)$ is finite (see section 5 ).

The notion of "relative angle" $\beta_{v}$ at a vertex will turn out to be crucial in the rest of this study.

Definition 2 For each vertex $v \in \widetilde{V}$, let $l_{v}$ be its valence, i.e. the number of faces of $\widetilde{P}$ which are adjacent to $v$, and name such faces as $F_{i_{1}}$, $\ldots, F_{i_{l v}}$. Let $\alpha_{i_{j}}, 1 \leq j \leq i_{l_{v}}$, be the angle at $v$ corresponding to face $F_{i_{j}}$. The relative angle at $v$ is then defined as

$$
\beta_{v}=2 \pi-\sum_{j=1}^{l_{v}} \alpha_{i_{j}} .
$$

The relative angle at a vertex (see fig. 4) is also known in the literature as the curvature concentrated at the vertex. Note that $0<\beta_{v}<2 \pi$ since $\widetilde{P}$ is a convex polyhedron with null curvature on its faces. We also have the classical Euler relation given by

Proposition 1 (Euler relation) Let $\widetilde{P}$ be a convex polyhedron and $\widetilde{V}$ the set of its vertices. Then,

$$
\sum_{v \in \widetilde{V}} \beta_{v}=4 \pi .
$$

We state now two remarks that can also be seen as basic properties of the motion of a polyhedra on a plane. There is no proof because these remarks are elementary.

Remark 1 Let $v \in \widetilde{V}$ and suppose that $\widetilde{P}$ rests on $P$ on a face $F_{i}$ with $F_{i} v$. By rolling clockwise $\widetilde{P}$ on all the faces containing $v$ until coming back to $F_{i}$ while keeping $v$ immobile, $\widetilde{P}$ is rotated of an angle $2 \pi-\beta_{v}$ around an axis $Z_{v}$ orthogonal to $P$ and passing through $v$, i.e., it moves from $(x, y, v, \theta, i)$ to $\left(x, y, v, \theta+\beta_{v}, i\right)$. We denote this trip by $R_{\beta_{v}}$ and the analogous counterclockwise trip by $R_{-\beta_{v}}$. By repeating $R_{\beta_{v}}$ clockwise or counterclockwise, we can go from $(x, y, v, \theta, i)$ to $\left(x, y, v, \theta+n \beta_{v}, i\right), n \in \mathbf{Z}$.

If $\frac{\beta_{\bar{v}}}{\pi}$ is irrational, then $\left\{n \beta_{v}\right\}_{n \in \mathbf{Z}}$ is dense in $S^{1}$, that is

$$
\forall \varepsilon>0, \forall \psi \in S^{1}, \exists n \in \mathbf{Z}:\left|n \beta_{v}-\psi\right|<\varepsilon .
$$

We will refer to (5) as to the property of reorienting $\widetilde{P}$ "arbitrarily close" (AC for short) to any direction.

Remark 2 Suppose that a configuration $q_{1}=\left(x_{1}, y_{1}, v, \theta_{1}, i\right)$ is brought in $q_{1}^{\prime}=$ $\left(x_{1}^{\prime}, y_{1}^{\prime}, v^{\prime}, \theta_{1}^{\prime}, i^{\prime}\right)$ by a certain trip $T$. Then, applying $T$ to any configuration $q=(x, y, v, \theta, i)$, we end up at $q^{\prime}=\left(x^{\prime}, y^{\prime}, v^{\prime}, \theta^{\prime}, i^{\prime}\right)$, where

$$
\begin{aligned}
\left(x^{\prime}, y^{\prime}\right)= & (x, y)+ \\
& \exp \left(i\left(\theta-\theta_{1}\right)\right)\left(x_{1}^{\prime}-x_{1}, y_{1}^{\prime}-y_{1}\right), \\
\theta^{\prime}= & \theta_{1}^{\prime}+\left(\theta-\theta_{1}\right) .
\end{aligned}
$$

For $i=1, \ldots, l$, let $\widetilde{T}_{i}$ be the set of all the trips starting and finishing with $F_{i}$ in contact. For any choice of $m-1$ out of the $m$ vertices of $\widetilde{P}$, labeled as $v_{1}, \cdots, v_{m-1}$, the following property holds:

Proposition 2 For all trips $T \in \widetilde{T}_{i}$, there exist $m-1$ integers $\left(n_{i}\right)_{1 \leq i \leq m-1}$ such that the total variation of orientation along $T$ is given by:

$$
\Delta \theta_{\mid T}=\sum_{i=1}^{m-1} n_{i} \beta_{v_{i}}
$$

Proof. To each trip $T \in \widetilde{T}_{i}$, a closed continuous path $\gamma_{T}$ can be associated as follows. Let $T=F_{i} \cdots F_{j} F_{k} \cdots F_{i}$. For all pairs of adjacent faces $F_{j} F_{k}$ with the edge $e$ in common, take a continuous path $\gamma_{j k}$ starting from the center of $F_{j}$ and finishing at the center of $F_{k}$, that passes through the edge $e$ only and through no vertex. 
The path $\gamma_{T}$ is obtained by concatenating such $\gamma_{i j}$ for all pairs of successive faces in $T$.

The polyhedron $\widetilde{P}$ is topologically equivalent to a two-dimensional sphere $S^{2}$ and is associated to $\widetilde{P}$, a curvature function $K$ defined as follows ([Spivak, 1979]):

$$
K(x)= \begin{cases}0 & \text { if } x \in \widetilde{P} \backslash \widetilde{V} \\ \beta_{v_{i}} & \text { if } x=v_{i}, 1 \leq i \leq m .\end{cases}
$$

Let $\gamma_{1}, \cdots, \gamma_{m-1}$ be a homology basis of $\widetilde{P} \backslash \widetilde{V}$ $\left(=S^{2} \backslash \tilde{V}\right)\left(\left[\right.\right.$ Spivak, 1979]). Every path $\gamma_{T}$ is therefore homologous to

$$
\sum_{i=1}^{m-1} n_{i} \gamma_{i}, \quad n_{i} \in \mathbf{Z}, 1 \leq i \leq m-1 .
$$

Each $\gamma_{i}, 1 \leq i \leq m-1$, can be taken as a simple continuous closed curve on $\widetilde{P} \backslash \widetilde{V}$ enclosing only $v_{i}$ in one of the two connected components it defines. It is clear that any trip $T$ associated with such a $\gamma_{i}$ has the same effects on the polyhedron as the trip $R_{\beta_{v_{i}}}$. The variation of orientation along $\gamma_{T}$ is equal to (Gauss-Bonnet theorem)

$$
\Delta \theta_{\mid T}=\sum_{i=1}^{m-1} n_{i} \beta_{v_{i}} .
$$

\section{Density of the reachable set}

The question whether a trip exists that can bring a given polyhedron $\mathrm{AC}$ to any configuration in $\widetilde{M}$, can be answered completely in terms of the curvature of the vertices of the polyhedron:

Theorem 1 The set of reachable configurations of a polyhedron $\widetilde{P}$ is globally dense in $\widetilde{M}$ if and only if there exists a vertex $\bar{v}$ such that $\frac{\beta_{\bar{v}}}{\pi}$ is irrational.

\section{Proof.}

$(\Rightarrow)$ The proof of the "if" part is subdivided as follows:

$$
\begin{array}{ll}
\frac{\beta_{\bar{v}}}{\pi} \text { irrational } & \stackrel{(i i)}{\Rightarrow}(\mathbf{D M})_{i} \text { holds } \\
(\mathbf{D M})_{i} \text { holds } & \stackrel{(i)}{\Rightarrow}(\mathbf{D M}) \text { holds } .
\end{array}
$$

Proof of $(i)$ : By hypothesis, there exists a trip that brings the polyhedron $\mathrm{AC}$ to $\left(x^{\prime}, y^{\prime}, \theta^{\prime}, i_{1}\right)$ for some $i_{1}$. We want to show that a trip exists that approaches $\mathrm{AC}\left(x^{\prime}, y^{\prime}, \theta^{\prime}, i\right)$, for all $i$ 's.

Let $T$ be a trip that brings $\left(x_{1}, y_{1}, \theta_{1}, i_{1}\right)$ into $\left(x_{2}, y_{2}, \theta_{2}, i\right)$, for any fixed $i$. By hypothesis, we can go $\mathrm{AC}$ to $\left(x^{\prime}{ }_{1}, y^{\prime}{ }_{1}, \theta_{1}^{\prime}, i_{1}\right)$, where

$$
\begin{aligned}
\left(x_{1}^{\prime}, y_{1}^{\prime}\right)= & \left(x^{\prime}, y^{\prime}\right)- \\
& \exp \left(i\left(\theta^{\prime}-\theta_{2}\right)\right)\left(x_{2}-x_{1}, y_{2}-y_{1}\right), \\
\theta_{1}= & \theta^{\prime}-\left(\theta_{2}-\theta_{1}\right) .
\end{aligned}
$$

By remark 2, there exists a concatenation of trips that brings $\mathrm{AC}$ to $\left(x^{\prime}, y^{\prime}, \theta^{\prime}, i\right)$ from $\left(x_{2}, y_{2}, \theta_{2}, i\right)$.

Proof of $(i i)$ : Let $\bar{q}=(0,0, \bar{v}, 0, i)$ be the initial configuration. We want to show that if $\frac{\beta_{\bar{v}}}{\pi}$ is irrational, then $(\mathbf{D M})_{i}$ holds.

Let $\widetilde{R}_{\bar{v}, i}$ the subset of $\widetilde{M}$ defined as

$$
R_{\bar{v}, i}=\{q \in \bar{q} \mid q=(x, y, \bar{v}, \theta, i)\} .
$$

Let us prove that the projection of $\widetilde{R}_{\bar{v}, i}$ on $\mathbb{R}^{2} \times S^{1}$ is everywhere dense. By remark 1 , if some configuration $(x, y, \bar{v}, \theta, i)$ is reachable from $\bar{q}$, then for every $\psi \in S^{1}$, we can get AC to $(x, y, \bar{v}, \psi, i)$. Therefore, it is enough to prove that $\Pi_{1}\left(\widetilde{R}_{\bar{v}, i}\right)$ is everywhere dense. In turn, the previous property is a consequence of the following one:

$$
\begin{gathered}
\exists \delta>0, \forall q \in \widetilde{R}_{\bar{v}, i}, \\
B_{\delta}\left(\Pi_{1}(q)\right) \subset \operatorname{clos}\left(\Pi_{1}\left(\widetilde{R}_{\bar{v}, i}\right)\right),
\end{gathered}
$$

where $\operatorname{clos}($.$) stands for the set closure.$

In order to prove (7), let us consider a vertex $v \in \widetilde{V}$, different from $\bar{v}$ and such that $\frac{\beta_{v}}{\pi}$ is irrational. The existence of the vertex $v$ is insured by the Euler relation. From $\bar{q}$, we can surely reach a point $q_{0}=\left(x_{0}, y_{0}, v, \theta_{0}, i^{\prime}\right)$ where $\vec{w}=\left(x_{0}, y_{0}\right) \neq 0$. The trip steering $\bar{q}$ to $q_{0}$ is denoted $L$ and the reverse trip, $L^{-1}$.

Consider the trip $T_{\beta_{\bar{v}}, \beta_{v}}$ defined as

$$
L^{-1} R_{\beta_{v}} L R_{\beta_{\bar{v}}} \text {. }
$$


By remark 2, a simple computation shows that we reach from every point $q \in \widetilde{R}_{\bar{v}, i}$ a point $q^{\prime} \in$ $\widetilde{R}_{\bar{v}, i}$ such that $\Pi_{1}\left(q^{\prime}\right)=\Pi_{1}(q)+\vec{t}$, where

$$
\vec{t}=\exp \left(i \beta_{\bar{v}}\right)\left(1-\exp \left(i \beta_{v}\right)\right) \vec{w} .
$$

In equation (8) and in $T_{\beta_{\bar{v}}, \beta_{v}}, \beta_{\bar{v}}$ and $\beta_{v}$ can be replaced respectively by any of their multiples $m \beta_{\bar{v}}$ and $n \beta_{v}$, with $m, n \in \mathbf{Z}$.

Since $\frac{\beta_{\bar{v}}}{\pi}$ and $\frac{\beta_{v}}{\pi}$ are irrational, by remark 1 and by $(8)$, we can therefore translate $\Pi_{1}(q)$ with a vector $\mathrm{AC}$ to any element of the set

$$
\left\{\exp (i \theta)(1-\exp (i \psi)) \vec{w} \mid(\theta, \psi) \in S^{1} \times S^{1}\right\},
$$

that is an open disc of radius $2\|\vec{w}\|$. Therefore, (7) is proved.

$(\Leftarrow)$ Assume now that there exist a vertex $v$ and a face $F_{i}$ with $F_{i} v$ such that $(\mathbf{D M})_{i}$ holds. We will show (a) $\Rightarrow(\mathrm{c})$.

If $\frac{\beta_{v_{i}}}{\pi}$ is rational for $1 \leq i \leq m$, we have

$$
\beta_{v_{i}}=\frac{p_{i}}{q_{i}} \pi
$$

Let $q$ be the smallest common multiple of the $q_{i}$ 's. As a consequence of equation (6), the variation of orientation along $\gamma_{T}$ is a entire multiple of $\frac{\pi}{q}$. This is a contradiction with $(\mathbf{D M})_{i}$. Therefore there must exists $\bar{v} \in \widetilde{V}$ such that $\frac{\beta_{\bar{v}}}{\pi}$ is irrational. This ends the proof of Theorem 1.

\section{Rolling a die}

Upon examination, it is clear that the set of configurations of a unit cube $\widetilde{C}$, that are reachable from a given initial configuration $q_{0} \in \widetilde{M}$, is a discrete set. Taking for instance $q_{0}=$ $(0,0,0,1)$, we have

$$
\widetilde{R} \subset \mathbf{Z}^{2} \times \widetilde{O} \times \widetilde{F},
$$

where $\widetilde{O}=\left\{k \frac{\pi}{2}, k=0, \ldots, 3\right\}$. Let $(\vec{\imath}, \vec{\jmath})$ denote an orthonormal frame of $P$ that generates the square lattice determined by the motion of $\widetilde{C}$. If $\vec{k}=\vec{\imath} \wedge \vec{\jmath}$, then $(\vec{\imath}, \vec{\jmath}, \vec{k})$ is an orthonormal basis of $\mathbb{R}^{3}$.
Observe that, given any point $q \in \widetilde{M}$, there are 4 ET's, each of them corresponding to an edge of the face in contact with $P$. Furthermore, the restriction of these actions to $\widetilde{O} \times \widetilde{F}$ are well-defined as

$r_{1}$, the rotation with respect to $\vec{\jmath}$ of angle $\pi / 2$, $r_{2}$, the rotation with respect to $\vec{\imath}$ of angle $\pi / 2$, and their inverses $r_{1}^{-1}$ and $r_{2}^{-1}$. Since these actions can be undertaken at every point of $M$, $\widetilde{O} \times \widetilde{F}$ can be seen as the group $G$ generated by $r_{1}$ and $r_{2}$ with the composition as the multiplication law. One further representation of $\widetilde{M}$ is thus obtained as

$$
\widetilde{M}=\mathbf{Z}^{2} \times G .
$$

The group $G$ is the proper symmetry group of the cube $\widetilde{C}$ and has a simple description using the group $S_{3}$ of permutations of 3 elements. Let $\sigma \in S_{3}$ be represented by the triplet $(\sigma(\vec{\imath}), \sigma(\vec{\jmath}), \sigma(\vec{k}))$ and $\epsilon \in \mathbf{Z}_{2}^{3}$ by $\left(\epsilon_{1}, \epsilon_{2}, \epsilon_{3}\right)$, with $\epsilon_{i}^{2}=1$ for $i=1,2,3$, where $\mathbf{Z}_{2}$ denotes the multiplicative group $\{-1,1\}$. Introduce the semi-direct product

$$
\sigma \odot \epsilon=\left(\epsilon_{1} \sigma(\vec{\imath}), \epsilon_{2} \sigma(\vec{\jmath}), \epsilon_{3} \sigma(\vec{k})\right),
$$

and let $G_{1}=S_{3} \odot \mathbf{Z}_{2}^{3}$. Note that the elements of $G_{1}$ transform the orthonormal basis $(\vec{\imath}, \vec{\jmath}, \vec{k})$ to another basis that is still orthonormal, but possibly with a different handedness. Then, $G$ is the subgroup of $G_{1}$ of the elements transforming $(\vec{\imath}, \vec{\jmath}, \vec{k})$ to another basis with the same handedness, i.e. such that $\operatorname{det}(\sigma(\vec{\imath}), \sigma(\vec{\jmath}), \sigma(\vec{k}))=1$. An element of $\widetilde{M}$ for the cube in the representation (9), is written as $(m, n, g)$.

Define $A_{1}$ (resp. $A_{-1}$ ) as the subset of $G$ corresponding to a product of an even (resp. odd) number of ET's. Note that $A_{1}$ is a subgroup of $G$ and, by construction of the multiplication table of $G$, one gets card $\left(A_{1}\right)=12$. The subset $A_{-1}$ has the same cardinality of $A_{1}$, however it is not a subgroup. Starting from a given face, there is an element in $A_{1}$ (resp. $A_{-1}$ ) that brings any face of the cube in contact with $P$. 
All elements of $A_{1}$ act on the cube so that it ends up with only two possible orientations, differing by $\pi$. The latter sentence applies to $A_{-1}$ as well, but the set of possible final orientations under $A_{1}$ and $A_{-1}$ are disjoint.

Consider next the infinite group $\widetilde{G}=\mathbf{Z}^{2} \oplus G$ (isomorphic to $\widetilde{M}$ ) with the multiplication law "." defined by

$$
(m, n, g) \cdot\left(m^{\prime}, n^{\prime}, g^{\prime}\right)=\left(m+m^{\prime}, n+n^{\prime}, g \cdot g^{\prime}\right) .
$$

The group $\widetilde{G}$ is generated by the two ET $R_{1}=$ $\left(\vec{\imath}, r_{1}\right)$ and $R_{2}=\left(\vec{\jmath}, r_{2}\right)$, and acts transitively on $\widetilde{M}$.

Define for $q=(m, n, g) \in \widetilde{M}$ and $g^{\prime} \in G$ the homomorphisms $h_{1}$ and $h_{2}$ as

a) $h_{1}(q)=m+n \quad(\bmod 2)$;

b) $h_{2}\left(g^{\prime}\right)=1$ if $g^{\prime} \in A_{1}, \quad-1$ if $g^{\prime} \in A_{-1}$.

We are now in a position to state our main results concerning the problem of rolling a die on a plane. The first proposition makes explicit the lattice structure and the restrictions on the reachable set of configurations:

Proposition 3 Let $q=(m, n, g) \in \widetilde{M}$. Then

$$
\begin{aligned}
& \widetilde{R}_{q}=\left\{q^{\prime}=\left(m+m^{\prime}, n+n^{\prime}, g^{\prime}\right) \in \widetilde{M}\right. \\
& \text { with } \left.g^{\prime} \in A_{h_{1}\left(q^{\prime}\right) h_{2}(g)}\right\} .
\end{aligned}
$$

The second result deals with bounds on the number of maneuvers necessary to reach an arbitrary configuration in $\widetilde{R}_{q}$ :

Proposition 4 Every $q^{\prime}=\left(m^{\prime}, n^{\prime}, g^{\prime}\right) \in \widetilde{R}_{q}$ can be reached from $q=(m, n, g) \in \widetilde{M}$ by a trip of length $L$ with

$$
\begin{aligned}
L \geq & \left|m^{\prime}-m\right|+\left|n^{\prime}-n\right| \\
L \leq & \left|m^{\prime}-m\right|+\left|n^{\prime}-n\right|+6 \\
L \leq & \sup \left\{4,\left|m^{\prime}-m\right|\right\}+ \\
& \sup \left\{4+\left|n^{\prime}-n\right|\right\}
\end{aligned}
$$

The proof of these propositions is based on examination of the multiplication table of the group $G$ above defined, and is omitted for brevity.

Remark The lower and upper bounds (10) and (12) coincide in the region $\left|m^{\prime}-m\right| \geq 4$, $\left|n^{\prime}-n\right| \geq 4$. An optimal trip therefore exists for any reachable configuration in this region, and its length is exactly $\left|m^{\prime}-m\right|+\left|n^{\prime}-n\right|$.

\section{Conclusions}

In this paper we undertook the analysis of the set of configurations that a polyhedron can be brought to reach by rolling on a plane about its edges. The problem appears to be important to practical applications, such as that of automatic part manipulation, as well as theoretically stimulating. As a result of our analysis, we pointed out that the structure of the reachable set may show a much richer variety for different polyhedra than it results for different regular surfaces, which were analyzed previously. Results of this paper concern only two extreme cases, while several intermediate cases with different characteristics were not solved here (for instance, a right-angled box with different sides).

Among the many open problems that are left for future work, we point out that the criterion for density of the reachable set of Theorem 1 is based on the irrationality of a quantity, whose actual value in physical problems can only be determined up to an error. It is clear therefore that it would be important to have more robust measures of how "manipulable" a given polyhedron is.

\section{References}

Aiyama, Y., Inaba, M., and Inoue, H.: "Pivoting: a new method of graspless manipulation of object by robot fingers", Proc. IEEE/RSJ Int. Conf. on Int. Robots and Systems, IROS'93, 1993.

Bicchi, A., Prattichizzo, D., and Sastry, S.S.: "Planning motions of rolling surfaces", Proc. IEEE Conf. on Decision and Control, 1995.

Bicchi, A., and Sorrentino, R.: "Dexterous manipulation through rolling", Proc. IEEE Int. Conf. on Robotics and Automation, pp. 452457, 1995.

Chen, I-M., and Burdick, J.W.: "A qualitative test for n-finger force-closure grasps on planar objects with applications to manipulation 
and finger gaits", Proc. IEEE Int. Conf. on Robotics and Automation, pp. 814-820, 1993.

Erdmann, M.A., Mason, M.T., Vanvevcek, G.: "Mechanical parts orienting: the case of a polyhedron on a table", Algorithmica, vol. 10, no.2, 1993.

Goldberg, K.: "Feeding and sorting algorithms for the parallel -jaw gripper", Proc. 6-th Int. Symposium on Robotics Research, 1993.

Jacobsen, S.C., Meek, S.G., and Fullmer, R.R.: "An adaptive myoelectric filter", 6th IEEE Conf. Engineering in Med. \& Biol. Soc., 1984a.

Li, Z., and Canny, J.: "Motion of two rigid bodies with rolling constraint", IEEE Trans. on Robotics and Automation, vol. 6, no.1, pp. 62-72, 1990.

Liu, Y., and Popplestone, R.: "A group theoretic formalization of surface contact", Int. J. of Robotics Research, vol. 13, no. 2, pp. 148161, 1994.

Lynch, K. M., and Mason, M. T.: "Pulling by pushing, slip with infinite friction, and perfectly rough surfaces", Int. J. of Robotics Research, vol. 14, no. 2, pp. 174-183, 1995.

Peshkin, M.A., and Sanderson, A.C.: "Planning robotic manipulation strategies for workpieces that slide", IEEE Journal of Robotics and Automation, vol. 4, no. 5, pp. , 1988.

Rus, D.: "Dexterous rotations of polyhedra", Proc. IEEE Int. Conf. on Robotics and Automation, pp. 2758-2763, 1992.

Salisbury, J.K., Brock, D.L., Chiu, S.L.: "Integrated language, sensing and control for a robot hand", Proc. $3^{\text {rd }}$ ISRR, Gouvieux, France, MIT Press, Cambridge MA, 1985.

Sawasaki, N., Inaba. M., and Inoue, H.: "Tumbling objects using a multifingered robot", Proc. 20th ISIR, pg. 609-616, 1989.

Spivak, M.: "Differential geometry", Publish or Perish, Houston, TX, 1979.
Tournassoud, P., Lozano-Perez, T., and Mazer, E.: "Regrasping", Proc. IEEE Int. Conf. on Robotics and Automation, pp. 1924-1928, 1987. 